\title{
What are GPs' views on the management of adolescents with mental health disorders? A qualitative study
}

\author{
Sagar Jobanputra* and Surinder Singh \\ Department of Primary Care and Population Health, University College London, \\ London, England
}

*Sagar Jobanputra, Medical Student, University College London, email address: sagar.jobanputra.15@ucl.ac.uk

\begin{abstract}
One in 10 children aged 11 to 16 years' have a mental health disorder. Whilst general practitioners (GPs) are considered ideal candidates to intervene and provide support, little research has focused on how this is managed, and the challenges they face, in the U.K. The aim of this work was to explore GPs' views on the management of adolescents with mental health disorders, and identify challenges they may face in current care. Eight GPs working in London were interviewed, using a semi-structured topic guide and a pre-prepared structured vignette. GPs with more clinical experience felt greater confidence in offering an intuitive approach to care. Many emphasised the therapeutic value of partnership with adolescents and discussed the complexity of identifying and labelling adolescents with a mental health disorder. Most GPs highlighted the need for a social picture: assessing their level of socio-economic deprivation, and the impact of parents. However, through this focus, some GPs felt adolescents from middle class backgrounds could be overlooked. This study offers some insight into the considerable impact GPs have on adolescents with mental health disorders, and challenges they face. To meet these challenges, proposals include a named contact within secondary care, and improved resources.
\end{abstract}




\section{What are GPs' views on the management of adolescents with mental health disorders? A qualitative study}

\section{Context}

With 1 in 10 children aged 11-16 suffering from a mental health disorder ${ }^{1}$, the prevalence of adolescents with mental health disorders is significant, and consequential impacts alarming. Evidence has shown that general practitioners (GPs) form an ideal position to intervene, and support these individuals ${ }^{2}$, yet little research has focused on how this is managed, and the challenges GPs may face, in the U.K.

\section{Aim}

To explore GPs' views on the management of adolescents with mental health disorders, and identify challenges they may face in current care.

${ }^{1}$ Hazel, G. et al. (2005). Mental health of children and young people in Great Britain, 2004. [online] Norwich: National Statistics, p.8. Available at: https://files.digital.nhs.uk/publicationimport/pub06xxx/pub06116/ment-heal-chil-youn-peopgb-2004-rep2.pdf [Accessed 12 Oct. 2019].

${ }^{2}$ O'Brien, D. et al. (2016) 'Barriers to managing child and adolescent mental health problems: a systematic review of primary care practitioners' perceptions.', The British Journal of General Practice (BJGP): The Journal of the Royal College of General Practitioners. British Journal of General Practice, 66(651), pp. e693-707. doi: 10.3399/bjgp16X687061 


\section{Study Description}

- Eight GPs were recruited via convenience sampling, from two practices within the boroughs of Camden and Haringey, London. One-to-one, semi-structured interviews were conducted, using a topic guide and a structured vignette which had been pre-prepared.

- The topic guide explored GPs' experiences of managing adolescents with mental health disorders, whilst the structured vignette gave GPs the opportunity to discuss options for management, and their awareness of current services.

- Interviews were audio-recorded and subsequently transcribed verbatim for thematic analysis. The transcription of interviews, and initial coding, were conducted by the student researcher then reviewed alongside SS.

- A constant comparative method of reviewing sequential interviews helped to inform further iterations of the topic guide.

\section{Outcomes}

Of the eight GPs interviewed, six were female and two male. The majority of GPs were salaried GPs, with one GP partner and one academic ST3 trainee.

Four key themes were identified: experience, partnership, identifying an adolescent with a mental health disorder and the social picture of adolescents. 


\section{Theme 1: Experience}

GPs involved in this study had accrued a range of clinical years' experience - from two to nineteen. Those with more years of clinical experience tended to employ an intuitive approach to care, with less reliance on resources, so felt more confident in managing an adolescent with a mental health disorder. In contrast, recently qualified GPs highlighted a lack of applicable training in primary care, which led to a more tentative approach. In order to supplement their clinical experience, some GPs drew upon personal experience as a parent, and as an adolescent themselves, to aid the consultation.

\section{Theme 2: Partnership}

GPs encouraged adolescents to present independently, giving scope to build rapport and form a partnership. Through this, some GPs went on to note its 'therapeutic impact'. However, difficulties in accessing secondary care, with lengthy waiting times and a discord in communication, impacted the support GPs could provide, thereby straining this partnership.

\section{Theme 3: Identifying an adolescent with a mental health disorder}

Many GPs noted the challenge of distinguishing 'normal' from 'abnormal' behaviour, particularly as these distinctions are often incorrect. Many practitioners thought the use of a diagnostic tool to facilitate this distinction would ingrain a 'tick-box approach' to care, so questioned its predictive validity. However, some practitioners noted that a diagnostic tool could provide a sensitive, neutral approach to disorders they were concerned of making worse by drawing reference to, such as eating disorders. The impact of the label itself was also widely discussed. 
Whilst this was met with apprehension by many practitioners, some noted the positive aspects of a label; for example, it could provide a context for an adolescent to frame their feelings and thoughts.

\section{Theme 4: The social picture}

Socio-economic deprivation as a risk factor was highlighted by several GPs, due to its wide impact on presentation and management. However, some practitioners noted how this focus could hinder support for adolescents from middle class backgrounds, who could be overlooked.

Parents also formed a key part of this social picture - either positively or negatively. Whilst some practitioners highlighted the value of a corroborative narrative to an adolescent's social health, many noted how this involvement could become detrimental - especially where relationships were fractured.

\section{Conclusions}

With the sheer number of adolescents living with mental health disorders, there is a demonstrable need for effective support. This qualitative study has begun to highlight the potential impact GPs can make, in light of just some of the barriers that they face. To address these challenges, proposals included a named contact within secondary care to help co-ordinate care, and wider distribution and integration of resources in training.

It is important to note the study did not reach theoretical saturation and was limited by its range of access to available practitioners. 
Approaching GPs earlier in the recruitment process, particularly trainees, would have helped to generate a more inclusive insight into the views of GPs on the management of adolescents with mental health disorders.

Overall, this project has provided a valuable insight into the field of qualitative research, from its initial conception, to its analysis and publication. Through this journey, I have gained a greater appreciation of how GPs navigate the intricacies of adolescent care, and just some of the challenges they face.

\section{Ethical Approval}

Approval for the study was confirmed by the NHS London Surrey Borders Research Ethics Committee (reference number: 18/LO/0278), and NHS Health Research Authority (IRAS project ID: 240866) on 16 February 2018, and 7 March 2018, respectively. A minor amendment to include the borough of Camden as a research site was confirmed on 14 March 2018.

Word Count: 818 